\title{
Lean Construction, como os princípios do Sistema Toyota de Produção podem contribuir para construções mais enxutas, produtivas e sustentáveis: Um estudo de caso na construtora Andrade Gutierrez.
}

\author{
Lean Construction, how the principles from Toyota's Production System can
} contribute developing constructions that are leaner, more pruductable and sustainable. A case study at Andrade Gutierrez.

\author{
Matheus Freire Silva ${ }^{1}$ \\ Eugênio Batista Leite ${ }^{2}$ \\ Gláucia Alves da Costa ${ }^{3}$ \\ Nayara de Oliveira Faria ${ }^{4}$ \\ Scarlet Marraine Gomes da Rocha ${ }^{5}$
}

\section{Resumo}

A baixa produtividade presente na construção civil, aliada a carência de mão de obra qualificada, tem dificultado cada vez mais o desenvolvimento do setor de construção civil no Brasil e no mundo. Diante disso, vários prejuízos são observados, destacando-se: falhas de planejamento, que acarretam interrupções em processos e retrabalhos; maior tempo de entrega da obra, aumentando o custo final do empreendimento; e desperdícios, que causam danos negativos, tanto economicamente, quanto ambientalmente. Dessa forma, constatou-se que diversos fatores prejudicam a evolução do setor de maneira sustentável e econômica, dificultando que haja um fluxo contínuo na produção. Existem princípios, metodologias e filosofias que podem evitar esses prejuízos e que viabilizam o crescimento do setor, permitindo maior produtividade, sustentabilidade e otimização de seus

\footnotetext{
Artigo recebido em 31 de Maio de 2017 e aprovado em 05 de Novembro de 2018

${ }^{1}$ Graduando em Engenharia Civil, pela Pontifícia Universidade Católica de Minas Gerais. Brasil. E-mail: freirematheuseng@gmail.com;

${ }^{2}$ Mestre em Educação pela Pontifícia Universidade Católica de Minas Gerais (PUCMG), graduado em Ciências Biológicas e Odontologia pela Pontifícia Universidade Católica de Minas Gerais (PUCMG). Pró Reitor Adjunto e Professor Adjunto do Departamento de Ciências Biológicas da Pontifícia Universidade Católica de Minas Gerais em Betim (PUCMG). Brasil. E-mail: eugeniobl@ pucminas.br;

3 Mestre em Engenharia de Produção pela Universidade Federal do Rio de Janeiro (UFRJ), com especialização em Gestão e Inovação. MBA - Gestão e Administração de Negócios, Estratégia pela Universidade Federal do Rio de Janeiro (UFRJ). Graduada em Engenharia Civil pela Universidade Federal de Minas Gerais (UFMG). Brasil. E-mail: glaucia.alves@agnet.com.br;

${ }^{4}$ Graduada em Engenharia de Produção, pela Pontifícia Universidade Católica de Minas Gerais (PUCMG). Brasil E-mail: nayaraoliveirafaria@gmail.com;

${ }^{5}$ Graduanda em Engenharia Civil, pela Pontifícia Universidade Católica de Minas Gerais (PUCMG) Brasil. E-mail: scarletmarraine@ hotmail.com.
} 
processos. Essas metodologias têm sido cada vez mais procuradas e implementadas, principalmente pelas grandes empresas. Portanto, essa pesquisa tem como objetivo analisar os princípios da filosofia Lean e como ela pode ser implementada na construção civil, a fim de otimizar e desenvolver o setor. A pesquisa é do tipo descritiva e os resultados tanto quantitativos, quanto qualitativos da utilização da filosofia Lean Construction poderão ser observados em um estudo de caso realizado na construtora Andrade Gutierrez, destacam-se o aumento da produtividade, redução de desperdícios e engajamento de pessoal.

Palavras-chave: Construção Civil. Lean Construction. Produtividade . Sustentabilidade.

\begin{abstract}
The low productivity that has been present in civil construction for the lasts years, allied with the lack of qualified labor, has been an obstacle in the sector's further development, not only in Brazil, but also worldwide. Therefore, the production chain faces many sorts of harms, highlighting: planning failures, which forces processes' interruptions and reworks; a delay of the construction's deadline, enlarging the final cost of the enterprise; and wastes, the cause of both economic and environmental damage. In that way, it has been noted that many factors work in detriment of a sustainable and economic unfolding of the area, making a continuous production flow difficult. There are, nevertheless, principles, methodologies and philosophies that can avoid those sources of damage, making feasible the sector's growth, allowing more productivity, sustainability and optimization of its processes. Those methodologies have been increasingly requested and implemented, especially by large companies. Thus, this paper aims to analyze the principles from the Lean philosophy and how it is possible to apply them in civil construction, as a mean to optimize and develop the sector. This research is characterized as descriptive with both quantitative and qualitative results. Results for the current paper were obtained through a case study carried out at the construction company Andrade Gutierrez with the main focus on the application of the Lean Construction philosophy in the company, highlighting the increase of productivity, reduction of waste and personnel engagement.
\end{abstract}

Keywords: Civil Construction. Lean Construction. Productivity. Sustainability.

\title{
1 Introdução
}

O setor da construção civil no Brasil cresceu de forma expressiva nos últimos dez anos. De acordo com Barreiros et al. (2014), entre os anos de 2007 a 2012, o crescimento do Produto Interno Bruto (PIB) deste segmento foi 1,8 vezes maior do que o PIB geral do país. Adicionalmente, os custos deste setor cresceram a taxas muito elevadas, chegando a aproximadamente $60 \%$ ao ano, ocasionando a redução da margem EBITDA $^{6}$ do negócio,

\footnotetext{
${ }^{6}$ EBITDA (Earning Before Interests, Taxes, Depreciation and Amortization), Lucro antes de juros, impostos, depreciação e amortização. Indicador financeiro utilizado pelas empresas de capital aberto.
} 
que caiu de $21 \%$ em 2007 para 16\% em 2011. Assim, a questão de aumento de produtividade no setor de construção civil começou a tomar uma relevância considerável.

De fato, quando comparado aos demais setores produtivos, a indústria da construção civil possui desenvolvimento e performance significativamente inferiores, apresentando “altos níveis de perdas e desperdícios, baixa produtividade, operações com elevados índices de incertezas, qualidade dos produtos aquém das expectativas e um grande número de acidentes de trabalho." (SOUZA, 2001, p.1).

De acordo com Cardoso, Detro e Canciglieri Júnior (2011), o Brasil consome cerca de uma tonelada de materiais de construção por metro quadrado edificado, produzindo níveis exorbitantes de desperdícios, o que deriva altos índices de resíduos e consequentemente degrada o meio ambiente. Sattler (2003), afirma que estimativas demonstram que a construção civil extrai cerca de 50\% dos recursos materiais da natureza. De maneira similar, dados demostram que este segmento gera no mundo $30 \%$ do lixo sólido, utiliza 20\% da água e 35\% da energia (CARDOSO; DETRO; CANCIGLIERI JÚNIOR, 2011).

Neste sentido, esforços capazes de reduzir a geração de resíduos e desperdícios, ao mesmo tempo em que se aumentam os níveis de produtividade e eficiência no setor civil, se fazem importantes e necessários. Portanto, começa-se a falar da aplicação do Lean manufacturing à construção civil. O termo Lean manufacturing, ou manufatura enxuta, foi introduzido por James Womack, Daniel Jones e Daniel Ross através do livro 'The machine that changed the world' (A máquina que mudou o mundo) lançado em 1990, que descreve o novo paradigma de manufatura estabelecido pelo Sistema Toyota de Produção (BERTANI, 2012). A manufatura enxuta está baseada nos princípios Lean Thinking, ou pensamento enxuto, que tem como prioridade a eliminação de qualquer atividade humana que absorve recursos sem a criação de valor (MARCHWINSKI, 2008). Bercaw (2012) salienta que a melhoria baseada em princípios Lean se sustenta em dois temas: melhoria contínua através da eliminação de atividades que não agregam valor, e respeito por pessoas. De maneira simples, melhorar um sistema baseando-se em princípios Lean, significa identificar todos os desperdícios a fim de eliminá-los (BERCAW, 2012). No que tange à construção civil, nove tipos de desperdícios são encontrados: espera, movimentação, 
processos desnecessários, área não utilizada, transporte, estoque, superprodução, defeitos e atrasos (HIRANO, 2009).

Em 1992, um finlandês chamado Lauri Kostela juntamente com a Universidade de Stanford, EUA, ligado ao CIFE - Center for Integrated Facility Engineering (Centro de Engenharia de Instalações Integradas), publicou um trabalho intitulado 'Application of the New Production Philosophy to Construction' (Aplicação da Nova Filosofia da Produção à Construção). O objetivo primordial da pesquisa era demonstrar os benefícios que o Sistema Toyota de Produção trouxe para linhas de produção da Toyota Motor Company, e como estes princípios poderiam beneficiar e serem aplicados ao setor da construção civil. Neste cenário, nasceu o conceito de Lean Construction, ou construção enxuta (MATIAS, 2012). Lean Construction é um termo adotado para designar a aplicação dos conceitos e princípios do Lean Manufacturing na construção civil (CÂMARA BRASILEIRA DA INDÚSTRIA DA CONSTRUÇÃO, 2012).

De acordo com Ross and Associates Environmental Consulting, Ltd. (2003), a aplicação de princípios Lean na construção cria um ambiente cultural e organizacional favorável para a diminuição de desperdícios e prevenção da poluição. Apesar de que estudos de casos e evidência empírica têm demonstrado que os benefícios ambientais decorrentes da aplicação das práticas Lean na construção civil são substanciais, raramente resíduos ambientais são caracterizados como os impulsionadores ou metas específicas para a implementação da filosofia Lean nas empresas deste setor. Ogunbiyi, Oladapo e Goulding (2014), conduziram um estudo de caso em diversas empresas de construção civil no Reino Unido que aplicam o Lean para uma construção mais sustentável. Dentre os benefícios encontrados pode-se destacar: melhoramento da imagem competitiva da organização e aumento da vantagem competitiva em sustentabilidade, melhoria de produtividade e do fluxo de produção, melhoria em qualidade do ambiente, cumprimento das expectativas dos clientes. Este mesmo estudo demonstrou que existe uma ligação direta entre a aplicação do Lean e a sustentabilidade, com a redução de desperdícios, gestão ambiental, maximização de valor, melhoria na saúde e segurança, etc. De maneira similar, estudos realizados por King e Lenox (2001), demonstram que a adoção de princípios Lean é capaz de reduzir não apenas os custos, como também os níveis de poluição e emissões de gases de efeito estufa 
em construções civis. De fato, o Lean é uma ferramenta poderosa para reduzir os impactos provindos de operações de transformação, ao mesmo tempo em que cria um "motor para uma melhoria contínua e sustentável do meio ambiente." (ROSS AND ASSOCIATES ENVIRONMENTAL CONSULTING, LTD., 2003, p. 21).

Em países desenvolvidos como os Estados Unidos, o conceito de Lean Construction já é bastante difundido e, portanto, várias construtoras trabalham com esta filosofia, como a DPR, Skanska e Bechtel. No cenário brasileiro poucas empresas de construção leve, localizadas no nordeste do país, utilizam-se deste conceito. Tratando do segmento de construção pesada, a primeira e única empresa identificada com esforços Lean, é a construtora Andrade Gutierrez (AG). A construção pesada tem processos que tendem a ser menos repetitivos do que a construção leve. Adicionalmente, este segmento é mais complexo e exige um planejamento diferenciado. Assim, por exemplo, um indicativo que corresponde ao ritmo de produção necessário para atender determinada demanda, chamado de planejamento takt, em um prédio com pavimentos é mais simples do que o planejamento takt de terraplanagem de uma hidrelétrica. Portanto, o desafio Lean para construções pesadas é mais complexo, e os conceitos desta filosofia devem ser adaptados para este nível maior de complexidade e particularidades. Diante disso, esta pesquisa tem como objetivo analisar os princípios da filosofia Lean e como ela pode ser implementada na construção civil, observando os resultados que a mesma trouxe para a construtora Andrade Gutierrez em um estudo de caso.

\section{Metodologia}

Considerando que o setor acadêmico se configura como um ambiente fértil para a implementação e análise de métodos e ferramentas Lean aplicados para a melhoria do setor da construção civil no Brasil, esta pesquisa se propõe a efetuar um estudo de caso do uso da filosofia Lean Construction na construtora Andrade Gutierrez (AG).

A pesquisa é do tipo descritiva, e foi desenvolvida com o método estudo de caso, muito utilizado em trabalhos de abordagem empírica, com investigação de fenômenos e processos que ocorrem dentro de um contexto real (MIGUEL, 2012). Uma das vantagens 
do uso desta técnica é que ela "possibilita uma análise mais sistêmica das situações encontradas.” (BERTUCCI, 2012, p. 53).

As informações foram coletadas através de entrevista realizada com a Superintendente em Excelência e Inovação da AG, Sra. Glaucia Alves, onde juntamente com buscas em dados publicados pela construtora, foi possível entender como funciona a filosofia Lean Construction dentro da construtora Andrade Gutierrez, quais ferramentas são utilizadas, boas práticas desenvolvidas, desafios enfrentados, e quais os benefícios que tal implementação resultou para a empresa.

\section{Lean Construction na Andrade Gutierrez}

\subsection{Histórico de implementação do Lean na AG}

A iniciativa de se implementar Lean no grupo Andrade Gutierrez surgiu a partir da obra do estádio da Amazônia para a Copa do Mundo em 2014. Este projeto estava envolto em grandes desafios e dificuldades. Primeiramente, a cidade sede do estádio, Manaus, está localizada no meio da floresta Amazônica, sendo acessível apenas através de barco ou avião, dificultando, portanto, a logística interna e externa da obra. O prazo de entrega do projeto era muito curto, e grandes modificações de escopo tiveram que ser feitas para cumprir exigências estabelecidas pela Federação Internacional de Futebol (FIFA), o que aumentou ainda mais o custo inicial do mesmo. Outro agravante foi a baixa qualificação de mão de obra da região e os altos níveis de turnover $^{7}(11,4 \%)$.

Neste cenário, a construtora enfrentava graves problemas de produtividade, e o aumento dos custos do projeto, ou a postergação da entrega do mesmo eram situações inviáveis com o cliente. Portanto, começou-se a procurar por iniciativas que possibilitassem o aumento da produtividade, redução dos custos e qualificação da mão de obra. Assim, contratou-se uma consultoria focada em lean manufacturing para auxiliar o grupo AG na

\footnotetext{
7 Turnover - No contexto de recursos humanos significa o movimento no qual um funcionário é substituído por um novo colaborador.
} 
implementação do Lean Construction como solução para os desafios e dificuldades que emergiram neste momento.

Primeiramente foi realizado todo replanejamento do projeto orientado aos processos críticos, com priorização de workshops em todas as áreas fabris da obra, central de armação, central de pré-moldados, central de concreto e central de carpintaria. O segundo passo consistiu em adaptar os novos conceitos para as frentes de produção, para a aplicação do Last Planner System, que até então não existia na empresa. O Last Planner System é a principal ferramenta Lean utilizada pela AG, e ela pode ser entendida como uma sistemática de planejamento focada em desdobramento contínuo e puxado do cronograma, com análise e eliminação de restrições antes da produção.

Segundo Ferreira (2010) o Last Planner System pode se entendido em três níveis hierárquicos: Um Plano Geral voltado ao longo prazo do projeto, um Plano de Antevisão, onde é observado o projeto em médio prazo e o Plano Semanal de Trabalho, contemplando o projeto em um curto prazo, conforme figura 1. Os planos de médio e curto prazos são os mais examinados no processo, pois a identificação de perdas nessas fases é mais acessível, pois os mesmos possuem mais detalhes.

Figura 1: Fases do planejamento Last Planner System

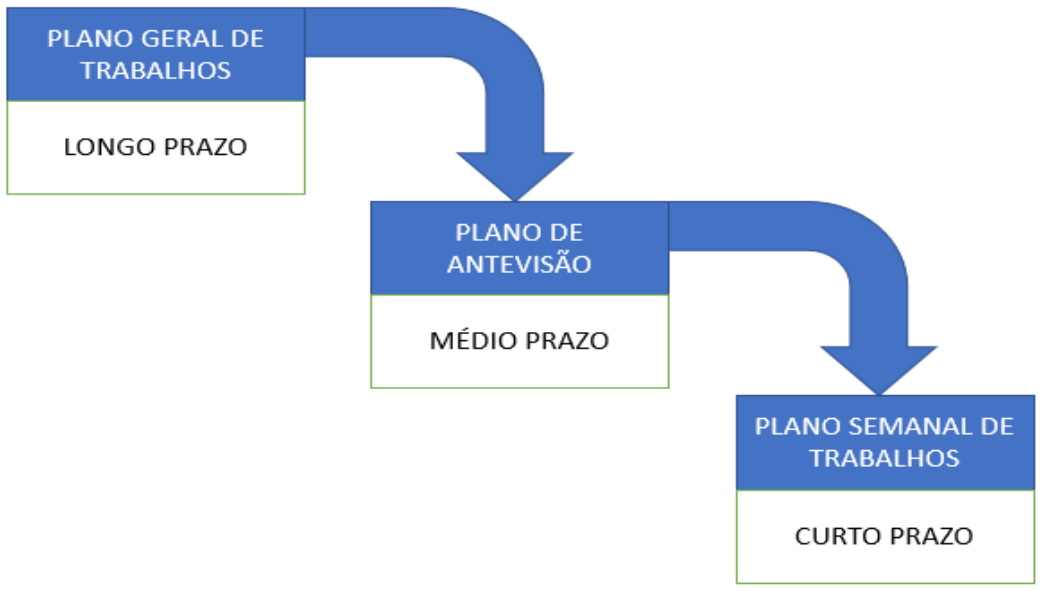

Fonte: Adaptado de Ferreira (2010)

Ainda de acordo com Ferreira (2010), o detalhamento possível utilizando a ferramenta Last Planner System é fundamental para o controle das atividades, além disso, 
as medições de desempenho são melhor analisadas já que os resultados podem observados semanalmente, podendo os processos serem otimizados e ajustados de maneira continua.

Os resultados da aplicação desta nova metodologia de trabalho foram significativos na $\mathrm{AG}$, com ganhos médios de produtividade da ordem de $22 \%$ a $23 \%$ para os principais processos aplicados, além de ganhos econômicos decorrentes. Tal performance foi o suficiente para que o Lean virasse uma diretriz estratégica do grupo Andrade Gutierrez.

\subsection{Lean no Cotidiano da AG}

Após o projeto do estádio em Amazonas, o grupo AG iniciou a implementação do Lean através de uma grande iniciativa estratégica chamada de Project One, que visava identificar, implementar e padronizar as melhores práticas de gestão nas obras e áreas de apoio, como engenharias e suprimentos, o projeto conta com 10 dimensões conforme figura 2 a seguir.

Figura 2: Dimensões do Projeto ONE

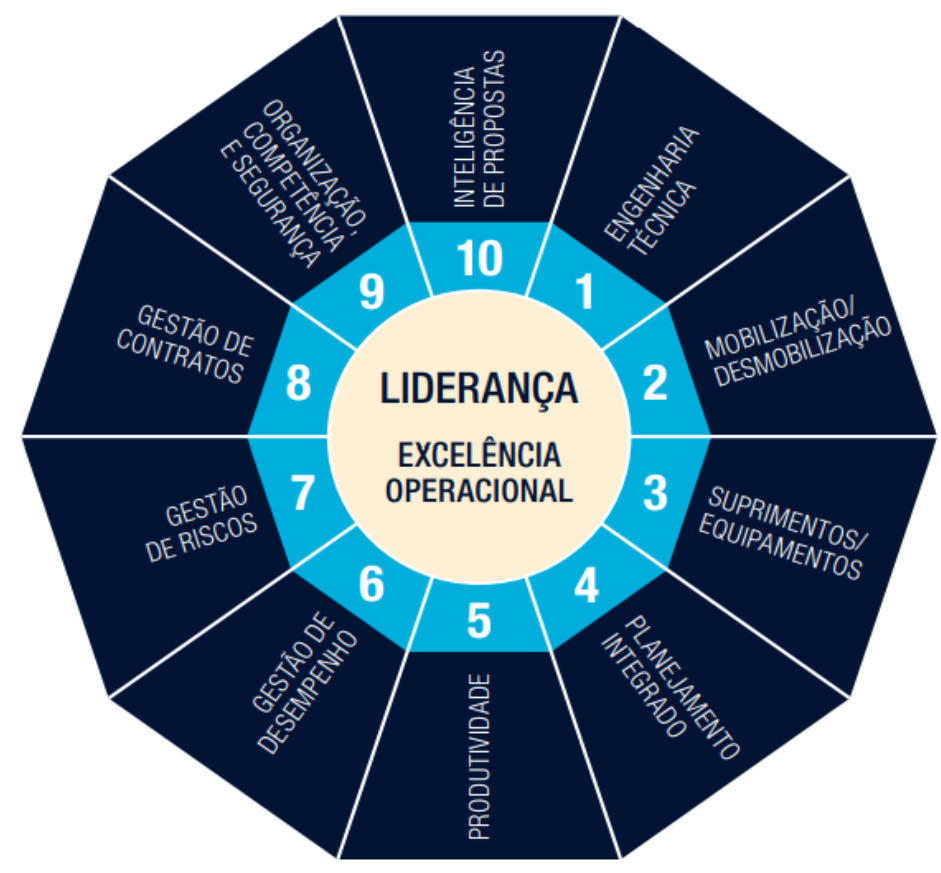

Fonte: Relatório de Sustentabilidade AG (2015, p. 37) 
Conforme publicado no Relatório de Sustentabilidade da Andrade Gutierrez (ANDRADE GUTIERREZ ENGENHARIA, 2015, p.37), com estas dimensões propõem-se atingir o objetivo final que é a padronização dos processos, com foco na melhoria contínua e eficiente, trazendo mais objetividade aos mesmos:

1. Engenharia Técnica: Eficiência na avaliação de projetos e busca de soluções para melhoria, gerando valor aos processos, da prospecção ao fim da execução;

2. Mobilização e Desmobilização: Metodização das movimentações com planejamento puxado;

3. Suprimentos e Equipamentos: Gestão de equipamentos, desde a compra até a logística;

4. Planejamento Integrado: Elaboração de cronogramas, dimensionamento de recursos e gestão do planejamento;

5. Produtividade: Acompanhamento e controle da produtividade das atividades;

6. Gestão de Desempenho: Monitoramento de performance, da obra até a presidência;

7. Gestão de Riscos: Identificação e mitigação de riscos;

8. Gestão de Contratos: Acompanhamento de marcos contratuais, gestão do relacionamento com o cliente, gestão de subempreiteiros;

9. Organização, Competências e Segurança: Estrutura de sistematização, ferramentas, ações preventivas de acidentes, desenvolvimento de pessoal;

10. Inteligência de Propostas: Formulação da proposta e interfaces entre propostas de orçamentação, áreas de apoio e execução da obra.

Após a implementação do Projeto One, foram alcançados diversos resultados positivos, destacando-se a capacitação para melhoria de produtividade de aproximadamente 6.000 colaboradores dos mais variados setores da empresa, e uma captura de valor estimada em R\$ 266 milhões. Este valor foi identificado através de um sistema estruturado de acompanhamento do valor capturado pelas implementações das dimensões.

Com os resultados expressivos alcançados economicamente, de engajamento de pessoal e principalmente na mudança de cultura da companhia, o Projeto One foi ampliado 
para as áreas corporativas e de negócios do grupo, formando o Sistema One de Excelência, abrangendo então todos os níveis e processos. O conceito do sistema está baseado em quatro princípios que contemplam integralmente os processos de gestão e operação, são eles: Excelência em clientes e negócios, Excelência em constrição, Excelência Corporativa, além de Pesquisa e desenvolvimento, conforme figura 3 a seguir.

Figura 3: Dimensões do Sistema One de Excelência

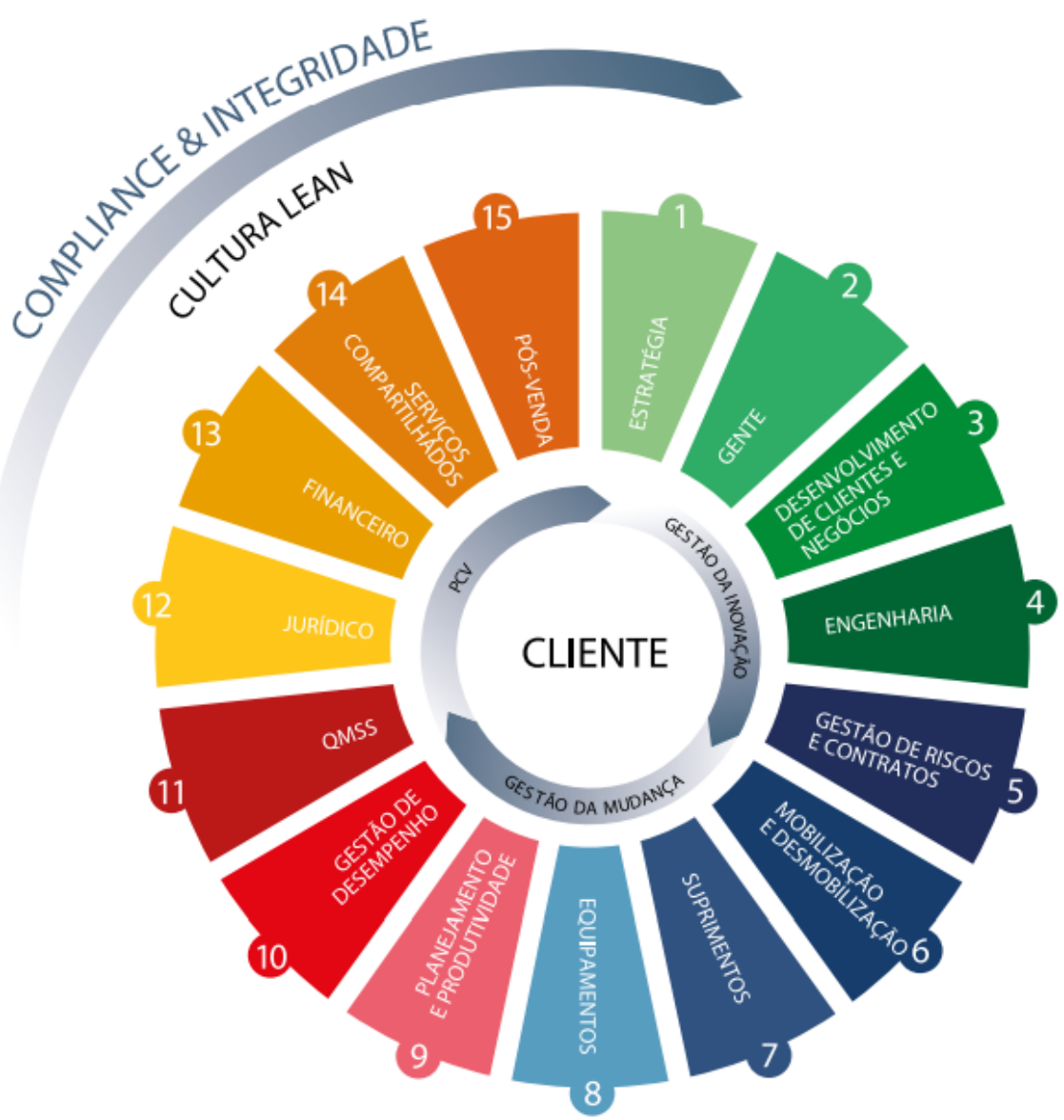

Fonte: Relatório de Sustentabilidade AG (2016, p. 44) 
As 15 dimensões que o sistema possui buscam interligar todos os processos da Companhia, elevando ao máximo o valor agregado para o cliente e evitando desperdícios e custos desnecessários, desde o estudo das oportunidades até o processo de pós-entrega e o encerramento dos contratos, bem como os processos corporativos e os relacionados ao desenvolvimento de clientes e negócios. O aumento na sinergia entre os setores, gera processos mais flexíveis e com a possibilidade de aprendizagem organizacional, proporcionando a reutilização de informações e soluções internas com ganhos de escala.

Como pode ser observado, a implementação da filosofia Lean traz uma visão holística sobre a maneira convencional do setor de construção civil, indo além do canteiro de obras e fazendo com que se torne uma cultura dentro da empresa, se a realização ocorre de maneira continua os benefícios são ainda mais elevados. Além de compreender a metodologia Lean Construction, é essencial que a implementação seja de uma forma sistêmica, para isso o conhecimento das ferramentas que possibilitarão a implementação deve ser bem fundamentado, observando as reais necessidades da demanda em questão, para assim, buscar a escolha das ferramentas que melhor se encaixam nos benefícios esperados com a utilização da filosofia (PADUA, 2013).

Segundo Fonseca Pinto (2012), a escolha pela metodologia Lean provoca um conjunto de mudanças para se atingir o objetivo final, dentre elas está a dedicação de uma serie de agentes de mudança que apliquem os conceitos da filosofia Lean, existem diversas ferramentas que auxiliam na aplicação, gestão, soluções e aferição dos resultados, ficando a escolha da empresa verificar quais delas poderão ser aplicadas e serão realmente funcionais para a demanda solicitada.

Diante disso, várias ferramentas provindas no Sistema Toyota de Produção hodiernamente são utilizadas na AG, como Andons, Heijunka Box, A3, 5W2H, SMED, Kanban, Milk Run, entre outros. Elas são as responsáveis por fazer com que a construção civil não apenas reduza os índices de desperdícios e custos em todas as fases da obra, como também fazem com que a obra tenha um fluxo contínuo, com um ritmo de produção balanceado e estável. Dentre as ferramentas destacam-se a ferramenta Heijunka, que é fundamental para que se consiga um nivelamento do ritmo dos trabalhos, a representação 
da diferença entre o ritmo convencional pode ser observada na figura 4, conforme Fonseca Pinto (2012).

Figura 4: Planejamento Heijunka

\begin{tabular}{|c|c|c|c|c|c|}
\hline \multicolumn{7}{|c|}{ PLANEJAMENTO CONVENCIONAL } \\
\hline & SEG & TER & QUA & QUI & SEX \\
\hline $09: 00$ & & & & & \\
\hline $10: 00$ & & & & & \\
\hline $11: 00$ & & & & & \\
\hline $12: 00$ & & & & & \\
\hline Almoço & & & & & \\
\hline $14: 00$ & & & & & \\
\hline $15: 00$ & & & & & \\
\hline $16: 00$ & & & & & \\
\hline $17: 00$ & & & & & \\
\hline $18: 00$ & & & & & \\
\hline
\end{tabular}

\begin{tabular}{|c|c|c|c|c|c|}
\hline \multicolumn{7}{|c|}{ PLANEJAMENTO HEIJUNKA } \\
\hline & SEG & TER & QUA & QUI & SEX \\
\hline $09: 00$ & & & & & \\
\hline $10: 00$ & & & & & \\
\hline $11: 00$ & & & & & \\
\hline $12: 00$ & & & & & \\
\hline Almoço & & & & & \\
\hline $14: 00$ & & & & & \\
\hline $15: 00$ & & & & & \\
\hline $16: 00$ & & & & & \\
\hline $17: 00$ & & & & & \\
\hline $18: 00$ & & & & & \\
\hline
\end{tabular}

\begin{tabular}{|l|l|l|}
\cline { 2 - 2 } \multicolumn{2}{c|}{} & CARGA DE TRABALHO \\
\hline \multirow{3}{*}{ Legenda } & & BAIXA \\
\cline { 2 - 3 } & & MEDIA \\
\cline { 2 - 3 } & & INTENSA \\
\hline
\end{tabular}

Fonte: Adaptado de Fonseca Pinto (2012)

No planejamento Heijunka a execução ocorre num ritmo constante durante toda a atividade. Esse é um grande problema na construção civil, algumas atividades convencionalmente não são subdivididas, gerando uma perca no tempo da execução, a dificuldade está em solucionar o problema de maneira que as atividades sejam niveladas e efetuadas de maneira eficiente desde o início e em um ritmo constante sem grandes variações, por isso esta ferramenta é importante nesse processo (Fonseca Pinto, 2012). Adicionalmente está a ferramenta Andon, que possibilita a capacidade de detecção de anormalidades no processo, ela conta com uma metodologia que envolve sinalizações com cores e efeitos sonoros que auxiliam na manutenção da execução das atividades.

Antes da aplicação de qualquer ferramenta, a filosofia da AG é dividir a obra em fatias (como fundações, estruturas e acabamentos) para que primeiramente o fluxo e o takt possam ser calculados e as atividades planejadas de forma balanceada, conforme figura 5 . Vale ressaltar que é muito difícil conseguir um takt unitário no segmento de construções 
pesadas, por isso a criação de uma hierarquia nos processos com a divisão em fatias é de grande valia.

Figura 5: Planejamento Takt em uma fachada

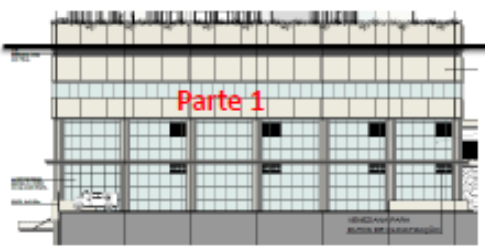

\begin{tabular}{|c|c|}
\hline \multirow{6}{*}{1} & Limpeza \\
\hline & Chapisco colante fachada \\
\hline & Chapisco fachada \\
\hline & Taliscamento fachada \\
\hline & Emboço 1 \\
\hline & Emboço 2 \\
\hline 2 & Cura \\
\hline \multirow{5}{*}{3} & Viga $2^{\circ}$ Pavimento \\
\hline & Chapisco colante / Emboço \\
\hline & Contramarco \\
\hline & Chumbamento \\
\hline & Elemento de concreto vazado \\
\hline 4 & Espala \\
\hline 5 & Granigliato \\
\hline \multirow{3}{*}{6} & Caixilho Pele de vidro \\
\hline & Vedação \\
\hline & Caixilho Brise \\
\hline
\end{tabular}

LEGENDA

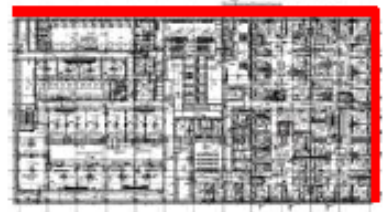

Fonte: Grupo AG (2016)

Outra ferramenta importante para otimização de processos é o Kanban, de acordo com Freitag (2015, apud SALEM et al. 2006) dos vários métodos que são associados ao just-in-time um dos principais é o kanban e está relacionado a um sistema de quadro com cartões, cestas ou sinais digitais e tem como objetivo reduzir os inventários, depósitos e/ou almoxarifados de acordo com pedidos vindo de trás no fluxo, essa ferramenta evita que haja um excesso de suprimentos ociosos armazenados e que ocupam áreas desnecessárias.

Em sua pesquisa, Mendes do Carmo (2014), apresenta um estudo de caso sobre a aplicação de ferramentas Lean para redução de desperdícios na construção civil, entre as ferramentas observadas está o kanban, no setor de almoxarifado foi observado que em horários como início de turnos para retirada de materiais formava-se fila, em alguns casos esse processo levava aproximadamente 1 hora e 30 minutos para que o ultimo colaborador fosse atendido. O desperdício identificado foi o tempo, para diminuição do mesmo foi implementado então a ferramenta, com ela os encarregados realizavam o pedido de material com antecedência para o próximo dia de serviço, dessa forma os responsáveis pela 
separação poderiam se programar e o material ficaria pronto para ser retirado no prazo correto, o resultado foi a redução e em alguns casos a eliminação das filas, aumentando a produtividade e reduzindo tal desperdício.

Apesar de não ser intuitivo, há uma certa quantidade de desperdício contido na movimentação durante execução de determinados serviços, de acordo com Barros (2013) o mapeamento das movimentações pode auxiliar na otimização dos processos, bem como determinar a localização de determinados setores no canteiro da obra. O procedimento é chamado de diagrama de Spaghetti e é simples de se realizar, consiste em traçar em uma planta baixa a movimentação de um colaborador ou produto no decorrer de um processo, com isso pode-se calcular a distância percorrida pelo colaborador ou produto observado e analisar maneiras de otimização, na figura 6 é possível visualizar um modelo do diagrama.

Figura 6: Modelo diagrama Spaghetti

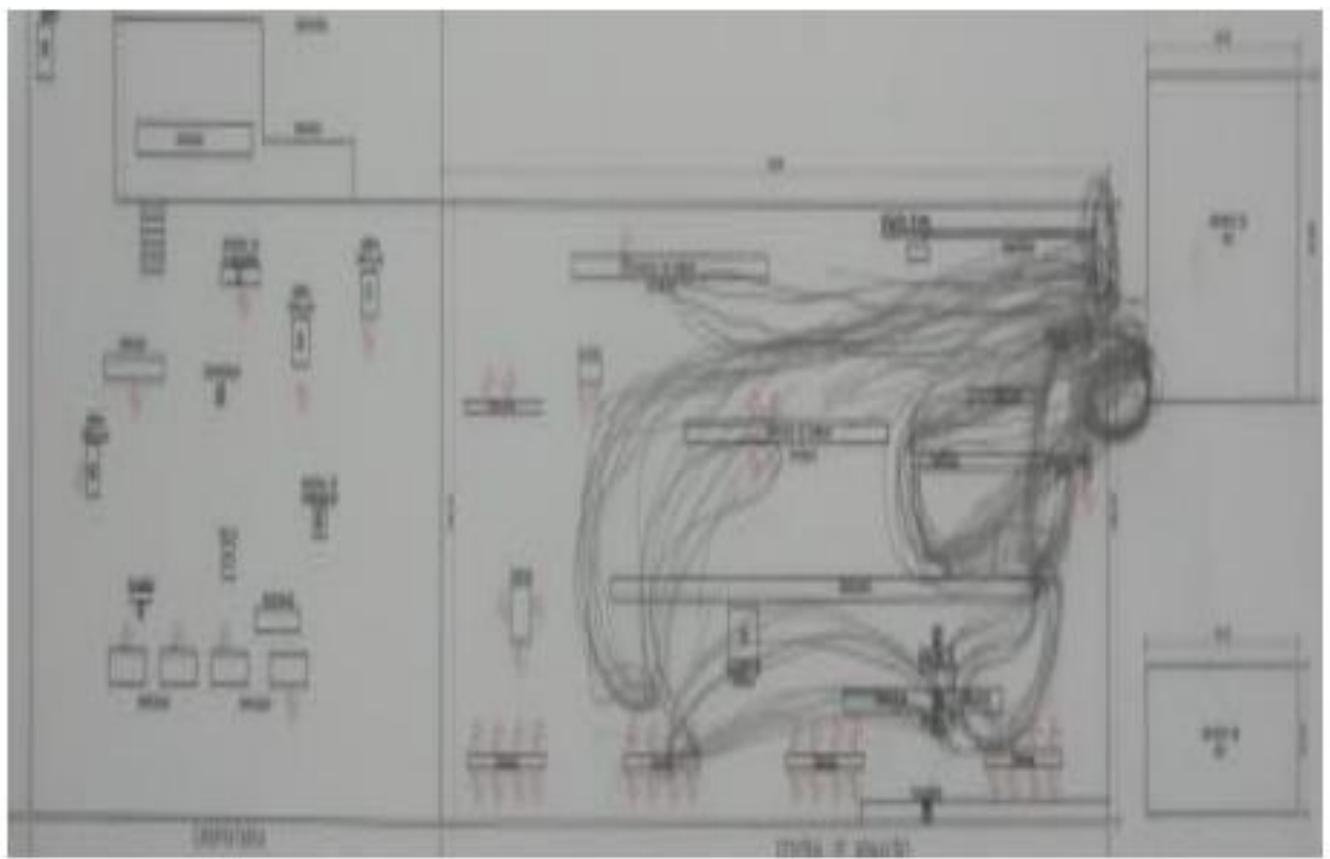

Fonte: Barros (2013) 
Para o grupo Andrade Gutierrez, além da importância que a ferramenta por si própria traz ao processo, sua credibilidade também é observada no processo como um todo. Para tanto, é essencial a plena participação de todos os envolvidos. Assim, a empresa investe consistentemente no treinamento de pessoal, com programas formais de treinamento e experimentação, coaching, mentoring e simulações, como, por exemplo, o Villego $^{8}$. Com isso se obtém também, uma padronização dos processos, o que possibilita aos colaboradores uma execução e gerenciamento de tarefas mais eficientes e com um domínio melhor sobre a função, essa padronização gera resultados mais estáveis, visto que quando se conhece os métodos e entende as etapas de determinada execução é possível diminuir os desperdícios e erros do processo (PADUA, 2013).

Adicionalmente, a AG possui programas de incentivo à execução e um programa de incentivo ao ownership ${ }^{9}$ do projeto. Deste modo, os colaboradores estão envolvidos durante todas as fases do planejamento e execução da obra, tendo uma visão sistêmica de todo projeto, se engajando mais na melhor execução do mesmo, e adquirindo certo nível de autonomia nas diversas etapas de execução da obra, reduzindo a burocracia comum de obras convencionais e tornando o projeto mais enxuto.

A AG possui um sistema de gestão da mudança para auxiliar a implantação da cultura Lean, chamado Influence Model, composto por quatro alavancas. A primeira é a alavanca do fazer sentido para as pessoas, ou seja, como a comunicação será feita e quais ferramentas de comunicação serão utilizadas para que as pessoas se sintam parte do processo de mudança. A segunda é a alavanca de capacitação, ou seja, como será o treinamento das pessoas, qual conteúdo elas precisam aprender, como será avaliado o aprendizado e como fazer com que a equipe de fato coloque em prática tudo que foi passado durante os treinamentos. A terceira alavanca diz respeito dos mecanismos formais ou afirmativos, ou seja, quais sistemas, estruturas e processos da empresa colaboram com os comportamentos que são esperados da parte dos funcionários, como moldar o ambiente de forma que ele garanta, ou ao menos incentive, a promoção de comportamentos corretos. A última alavanca está relacionada ao exemplo da liderança. O líder não pode simplesmente impor o que ele quiser com o Lean, ele tem que vivenciar a metodologia.

\footnotetext{
${ }^{8}$ Villego - Simulador para auxílio na utilização da ferramenta Last Planner System (LPS)

${ }^{9}$ Ownership - Termo referente a mentalidade diferenciada de quem almeja ver a organização prosperar, propiciando a ela crescimento, estabilidade e lucro
} 
Cabe a ele ter um bom quadro de gestão à vista, fazer as reuniões nos horários e nas pautas corretas, e cumprir todos os prazos e metas estabelecidas para a equipe. A AG identificou que se precisa de um modelo de gestão eficiente para transformar uma empresa com o Lean Construction. Neste modelo, tudo tem que ser o mais simples possível, se adequando, portanto, ao conceito enxuto.

Os indicadores de produção, planejamento, saúde e segurança, engenharia, produtividade, meio ambiente, gestão de recursos, financeiro, índices de satisfação dos clientes são acompanhados e supervisionados pela torre de controle. A torre de controle é um local no qual estes indicadores são colocados em gestão individual e visual. Reuniões periódicas de performance são realizadas em pé com o objetivo de que estas sejam o mais rápidas e objetivas possível. Adicionalmente, reuniões diárias de check-in e check-out são realizadas nas frentes de obra com o objetivo de acompanhar eficientemente o andamento da obra em cada setor, os possíveis problemas e desafios que estão surgindo no projeto e que poderão atrapalhar no sucesso do mesmo. As discussões propostas nas reuniões visam também a eliminação de tarefas com valor não agregado, já que as discussões diárias permitem a diminuição dos tempos de ciclo, fortemente ligada a eliminação de perdas, que estão principalmente nas etapas de movimentação, espera e inspeção (PADUA, 2013).

Na figura 7, Ferreira (2010) em seu estudo de caso realizado em Portugal, apresenta um esquema de como eram realizadas as reuniões de desempenho, controle e planejamento da obra acompanhada, as mesmas seguiam o cronograma estabelecido no Last Planner System. 
Figura 7: Reuniões de planejamento, análise e controle

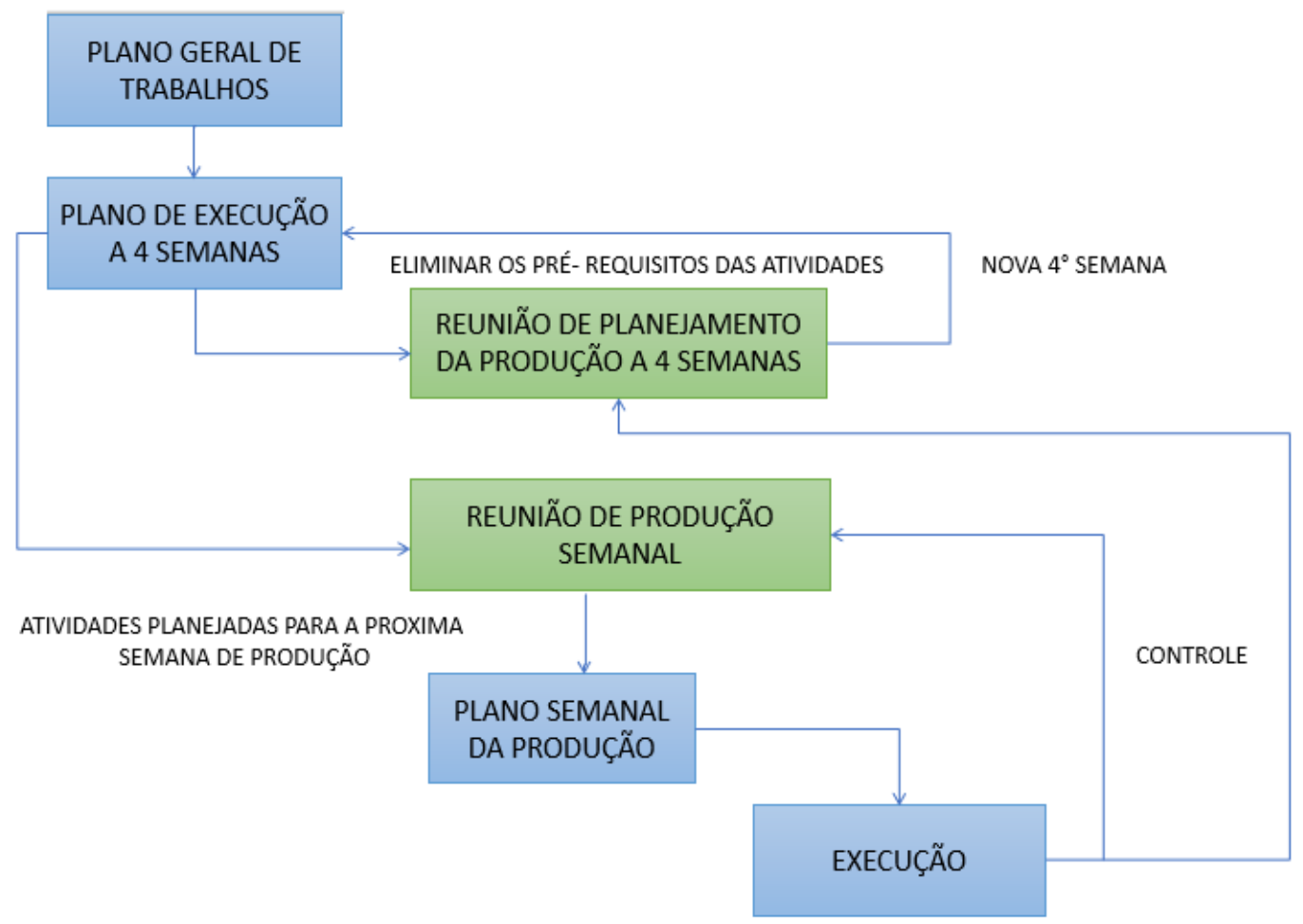

Fonte: Adaptado de Ferreira (2010)

Por fim, os principais atores responsáveis pelo sucesso do Lean na AG são os agentes de mudança. Agentes de mudança, ou ninjas, são pessoas que possuem alto poder de influência, boa comunicação, vasto conhecimento técnico de Lean, boa desenvoltura para falar, boas habilidades de negociação e liderança. A equipe é composta por 75 pessoas no Brasil, América Latina, Europa, África e Ásia, são eles os responsáveis em impulsionar e influenciar todos os demais funcionários para uma mudança organizacional voltada para a filosofia Lean Construction dentro da Andrade Gutierrez.

\section{Principais resultados alcançados com o Lean na AG}

Através da implementação da filosofia Lean Construction em suas obras, o grupo Andrade Gutierrez obteve resultados quantitativos e qualitativos significativamente expressivos. No que tange a produtividade, o aumento deste índice foi na ordem dos $20 \%$ para diversos processos dentro do canteiro de obras. Através da programação takt para construções pesadas, a empresa teve um ganho de $40 \%$ no tempo de execução da obra dos 
quatro pavimentos de internação do Hospital Metropolitano de Belo Horizonte (HMBH). Neste mesmo projeto, a redução dos índices de desperdícios foi na ordem de $20 \%$. Na figura 8 é possível visualizar como foi realizada a esquematização da aplicação do Lean na obra do Hospital Metropolitano de Belo Horizonte.

Figura 8: Lean na obra HMBH

\section{Aplicação Lean na Obra HMBH}

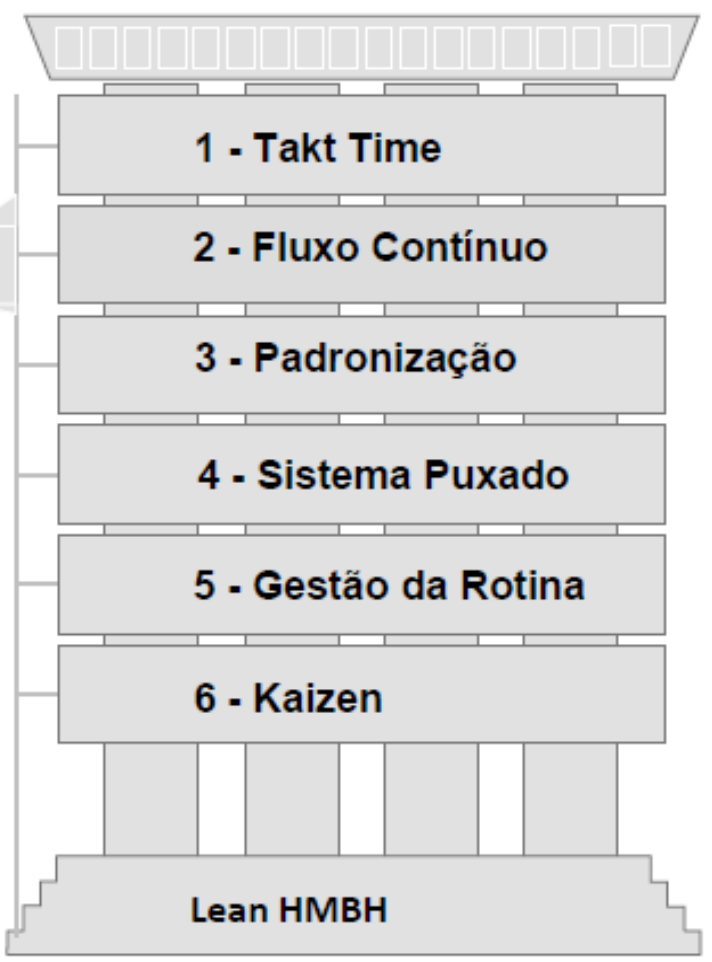

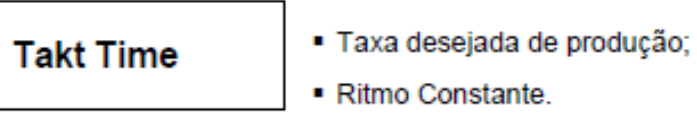

- Ritmo Constante.
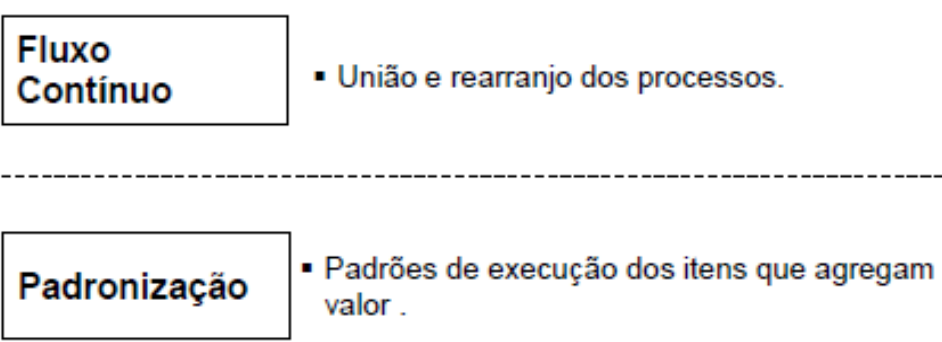

\begin{tabular}{|l|l}
\hline $\begin{array}{l}\text { Sistema } \\
\text { Puxado }\end{array}$ & $\begin{array}{l}\text { - Uma atividade antes da outra; } \\
\text { - Execução sobre demanda. }\end{array}$ \\
\hline
\end{tabular}

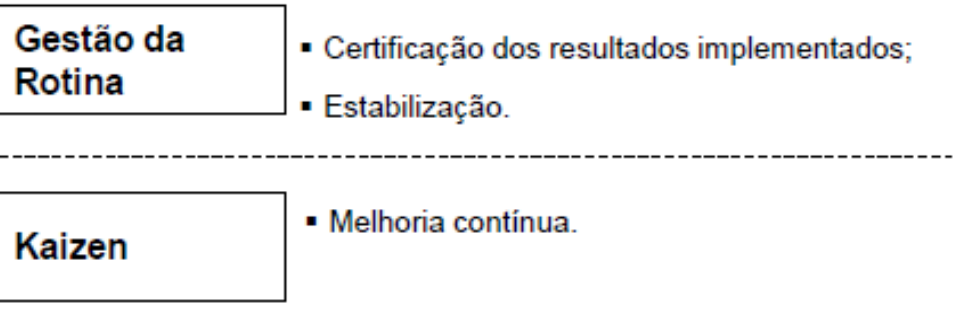

Fonte: Grupo AG (2016)

No projeto do estádio do Amazonas, através da otimização de layout, o índice de movimentação desnecessária dos operários foi reduzido em 87\%. Também, o índice de desperdício de materiais em áreas específicas, foi reduzido em 50\% (MATTOS et al. 2012).

Conforme divulgado através do Relatório de Sustentabilidade da Andrade Gutierrez (ANDRADE GUTIERREZ ENGENHARIA, 2016, p.39), o fruto do trabalho colaborativo, engajado pelos agentes de mudança através do Projeto One, gerou em 2015 uma captura de 
valor na ordem dos R\$115 milhões por meio "da geração de processos padronizados para as áreas mais críticas da empresa e capacitação de mais de 3 mil pessoas". Adicionalmente, através do mesmo projeto, o grupo já mapeou mais de $\mathrm{R} \$ 500$ milhões em oportunidades de melhorias.

Em termos qualitativos, o principal ganho está relacionado com a melhoria da cultura organizacional do grupo. A empresa atualmente conta com uma mão de obra mais bem qualificada, que entende e domina muito melhor todos os processos que envolvem o projeto de obra, exercendo o papel de problem solvers. Adicionalmente, os funcionários se tornaram mais flexíveis às mudanças, e a liderança tomou um papel mais participativo e menos diretivo. Também, os processos se tornaram mais transparentes, flexíveis, estruturados, padronizados, equilibrados e principalmente enxutos.

\section{Considerações finais}

Por meio desta pesquisa pode-se comprovar que, utilizando os princípios do Sistema Toyota de Produção aplicados à construção civil, se obtém eficientes resoluções de problemas que este setor enfrenta, como questões que envolvem a baixa produtividade, os altos níveis de desperdício, falta de padronização dos processos, altos custos, etc.

Em termos gerais, os princípios do Sistema Toyota de Produção através do Lean Construction contribuem para construções mais eficientes, produtivas e sustentáveis. Diante disso, podemos inferir que o Lean Construction contribui também para a redução dos impactos ambientais causados pela construção civil, uma vez que a redução de desperdícios atenua esses impactos negativos gerados pelo setor, contribuindo para que as obras se tornem mais sustentáveis.

Através do estudo de caso realizado, observou-se que com a implementação da filosofia Lean Construction na Construtora Andrade Gutierrez foi possível obter ganhos significativos, bem como, a padronização dos processos, aumento da produtividade, diminuição dos desperdícios, otimização nos planejamentos, e principalmente o desenvolvimento de pessoas envolvidas nos processos, tornando-as mais preparadas a solucionarem problemas e diminuição de erros. 
Porém, apesar dos significativos resultados demonstrados e da importância de novos métodos para desenvolvimento do setor da construção civil, a filosofia Lean Construction ainda é relativamente nova no Brasil e carece de novos estudos, exploração e efetividade, tornando nossas empresas mais produtivas, com processos mais enxutos, evitando desperdícios e trazendo competitividade no cenário nacional e mundial.

\section{Referências}

ANDRADE GUTIERREZ ENGENHARIA. Relatório de sustentabilidade 2015. Disponível em: <http://www.andradegutierrez.com/Imprensa.aspx\#674>. Acesso em: 20 set. 2016.

ANDRADE GUTIERREZ ENGENHARIA. Relatório de sustentabilidade 2016. Disponível em: < http://www.andradegutierrez.com.br/Imprensa.aspx\#722>. Acesso em: 20 set. 2016.

BARREIROS, F. et al. Estudo sobre produtividade na construção civil: desafios e tendências no Brasil. [S.1.]: Ernst \& Young, 2014. Disponível em: < http://www.ey.com/Publication/vwLUAssets/EY_Estudo_Produtividade_na_Construcao_C ivil/\$FILE/Estudo_Real_Estate.pdf >. Acesso em: 10 set. 2016.

BARROS, L. M. C. Estudo de técnicas para melhoria dos resultados de obras de infraestrutura mediante a adaptação e aplicação do sistema toyota de produção (Lean Production). Curso de Engenharia Civil da Escola Politécnica, Universidade Federal do Rio de Janeiro Rio de Janeiro, RJ - Brasil, Agosto de 2013

BERCAW, R. Taking improvement from assembly line to healthcare: the application of Lean within the healthcare industry. New York: CRC Press, 2012.

BERTANI, T. M. Lean healthcare: recomendações para implantações dos conceitos de produção enxuta em ambientes hospitalares. 2012. 166f. Dissertação (Mestrado) Programa de Pós-Graduação em Engenharia de Produção, Universidade de São Paulo, São Carlos, 2012. Disponível em: < file://C:/Users/877165/Downloads/Dissertacao_Thiago_Moreno_Bertani.pdf >. Acesso em: 16 set. 2016.

BERTUCCI, J. L. O. Metodologia básica para elaboração de trabalhos de conclusão de cursos (TCC): ênfase na elaboração de TCC de pós-graduação Lato Sensu. São Paulo: Atlas, 2012. 
CÂMARA BRASILEIRA DA INDÚSTRIA DA CONSTRUÇÃO. Núcleo de Meio Ambiente. C. Rolim Engenharia LTDA: Filosofia Lean. [Prêmio CBIC de Inovação e Sustentabilidade]. Fortaleza, 2012. Disponível em:

<file://C:/Users/877165/Downloads/FILOSOFIA\%20LEAN.pdf>. Acesso em: 20 set. 2016

CARDOSO, R. R.; DETRO, S. P.; CANCIGLIERI JÚNIOR, O. Uma visão tecnológica sobre o desenvolvimento de produtos e a sustentabilidade. In: CONGRESSO BRASILEIRO DE GESTÃO DE DESENVOLVIMENTO DE PRODUTO, 8., 2011, Porto Alegre. [Anais eletrônicos...]. Disponível em: < https://www.researchgate.net/publication/280882943_Uma_Visao_Tecnologica_sobre_o_D esenvolvimento_de_Produtos_e_a_Sustentabilidade >. Acesso em: 16 set. 2016.

CIDIN, R. C. P. J.; SILVA, R. S. Pegada ecológica: instrumento de avaliação dos impactos antrópicos no meio natural. Estudos geográficos, Rio Claro, v.2, n.1, p. 34-52, jun. 2004. Disponível em: < http://www.ifba.edu.br/professores/iarasantos/CA/PE/Pegada\%20ecologica_2004.pdf >. Acesso em: 16 set. 2016.

FONSECA PINTO, J.M. Lean Construction - Proposta de Metodologia de Avaliação de Projetos de Construção. FACULDADE DE ENGENHARIA DA UNIVERSIDADE DO PORTO, Portugal, Julho de 2012.

FERREIRA, R. Lean Constrution na Norlabor - Engenharia e Construção, SA. Porto: Feup, 2010.

FREITAG, A. E. B. Fatores críticos de sucesso para adoção da gestão "enxuta" pela indústria da construção civil do estado do Rio de Janeiro. Niteroi, RJ, 2015.

HIRANO, H. JIT Implementation manual: the complete guide to just-in-time manufacturing. 2nd. Vol. 1. Boca Raton. Productivity Press, 2009.

KING, A. A; LENOX, M. j. Lean and green? An empirical examination of the relationship between lean production and environmental performance. Production and operations management, v.10, n.3, fall 2001. Disponível em: < http://users.ipfw.edu/wellingj/ProductMix/publications/KingLenox.pdf >. Acesso em: 02 out. 2016.

MARCHWINSKI, C. (Ed.) Lean lexicon: a graphical glossary for lean thinkers. 4th. ed. Cambridge: Lean Enterprise Institute, 2008.

MATIAS, B. S. Introdução. In: UNIVERSIDADE FEDERAL DO CEARÁ. Programa de Educação Tutorial da Engenharia Civil. $1^{\text {a }}$ temporada de minicursos: Lean Construction. Ceará: UFC; PET Civil, 2012. Cap.1, p.3-5. Disponível em: < http://www.petcivil.ufc.br/portal/wp-content/uploads/2012/02/Lean-Cosntruction-v1.pdf > . Acesso em: 02 out. 2016. 
MATTOS, A. D. et al. The management challenges of building a world cup stadium in the Amazon - the case of the Amazon arena (Brazil). In: ICEC World Congres [International Fellow Award], 8., 2012, Durban, South Africa. Disponível em: < http://www.icoste.org/wp-content/uploads/2012/09/Management-Challenges-of-Buildinga-World-Cup-Stadium-in-the-Amazon.pdf>. Acesso em: 16 mar. 2017.

MENDES DO CARMO, B. S. Lean Construction: estudo de caso sobre a aplicação de ferramentas para a redução de desperdícios em empreendimento de construção civil. Pró-Reitoria de Graduação, Curso de Engenharia Civil, Trabalho de Conclusão de Curso, Brasília, DF, 2014.

MIGUEL, P. A. C. (Coord.). Metodologia de pesquisa em engenharia de produção e gestão de operações. 2. ed. Rio de Janeiro: Elsevier, 2012.

OGUNBIYI, O.; OLADAPO, A.; GOULDING, J. An empirical study of the impact of lean construction techniques on sustainable construction in the UK. Construction Innovation. v. 14, n. 1, p. 88-107, 2014.

PADUA, R. C. Implementação de Práticas de Lean Construction em uma Obra Residencial em Goiânia - Estudo de Caso. Universidade Federal de Goiás Escola de Engenharia Civil - Curso de Graduação em Engenharia Civil. Goiânia. 2013.

PONTIFICIA UNIVERSIDADE CATOLICA DE MINAS GERAIS. Pró-Reitoria de Graduação. Sistema Integrado De Bibliotecas. Orientações para elaboração de trabalhos técnicos científicos: projeto de pesquisa, teses, dissertações, monografias, relatório entre outros trabalhos acadêmicos, conforme a Associação Brasileira de Normas Técnicas (ABNT). 2. ed. Belo Horizonte: PUC Minas, 2016. Disponível em: <www.pucminas.br/biblioteca>. Acesso em: 18 mar. 2017.

\section{ROSS AND ASSOCIATES ENVIRONMENTAL CONSULTING, LTD. Lean}

manufacturing and the environment: research on advanced manufacturing systems and the environment and recommendations for leveraging better environmental performance. Oct. 2003. Disponível em: < https://www.epa.gov/sites/production/files/201310/documents/leanreport.pdf >. Acesso em: 10 set. 2016.

SANTOS, B. Construção enxuta com o emprego de ferramentas de gestão ágil: um estudo de caso. Instituto Federal de Educação Ciência e Tecnologia de Sergipe - IFS. Aracaju, 2017. $65 \mathrm{f}$.

SATTLER M. A. Edificações e comunidades sustentáveis: atividades em desenvolvimento no NORIE/UFRGS. In: SEMINÁRIO IBERO-AMERICANO DA REDE CYTED XIV, 4., 2002, São Paulo; Ros Mari Zenha (Coord.). Capacitação e transferência de tecnologia para habitação de interesse social: em busca de novas estratégias. São Paulo: IPT, 2003. 
v.2, p.219-229. Disponível em: <

http://www.habitare.org.br/pdf/publicacoes/arquivos/189.pdf >. Acesso em: 03 out. 2016.

SOUZA, E. A. A. O papel estratégico da manufatura: estudo de caso na indústria da construção civil. In: ENCONTRO NACIONAL DE ENGENHARIA DE PRODUÇÃO, 21.; INTERNATIONAL CONFERENCE ON INDUSTRIAL ENGINEERING AND OPERATIONS MANAGEMENT, 7., 2001, Salvador. Anais eletrônicos... Salvador: FTC, 2001. 1CD.

SOUZA, U. E. L. et al. Diagnóstico e combate à geração de resíduos na produção de obras de construção de edifícios: uma abordagem progressiva. Ambiente Construído, Porto Alegre, v. 4, n. 4, p. 33-46, out./dez. 2004. 